\title{
Interrupting the social amplification of risk process: a case study in collective emissions reduction
}

\author{
J.S. Busby*, R.E. Alcock, B.H. MacGilliuray \\ School of Management, Lancaster University, Lancaster LA1 4YX, UK
}

\section{A R T I C L E I N F O}

Published on line 8 January 2009

Keywords:

Social amplification

Emissions reduction

Brominated flame retardants

PBDEs

\begin{abstract}
A B S T R A C T
One of the main approaches we have for studying the progressive divergence of understandings around a risk issue is that of social risk amplification. This article describes a case study of a particular environmental contaminant, a chemical flame retardant that could be interpreted as having produced a risk amplifying process. It describes in particular how a group of industrial organizations acted collectively to reduce emissions of this contaminant, in an apparent attempt to avert regulation and boycotts-that is, to intercept the social amplification process and avoid its secondary effects. The aim of the study was to investigate the constitutive qualities of this collective action: the qualities that defined it and made it effective in the eyes of those involved. These include institutionalisation and independence, the ability to confer individual as well as collective benefit, the capacity to attract (rather than avoid) criticism, and the 'branding' that helps communicate what otherwise appear to be a set of unconnected, local actions. Although the risk amplification framework has been criticised for implying that there is some externally given risk level that is subsequently amplified, it does appear to capture the mentality of actors involved in issues of this kind. They talk and act as though they believe they are participants in a risk amplification process.
\end{abstract}

(C) 2008 Elsevier Ltd. All rights reserved.

\section{Introduction}

\subsection{A problem of risk 'amplification'}

This article is a case study of a flame retardant and environmental contaminant, decabromodiphenyl ether (Deca-BDE) that has been the object of significant controversy, advocacy group activity, lobbying and regulation. An extended risk assessment in Europe (ECB, 2002, 2004) found little positive evidence of harm, but admitted to being unable to determine on a scientific basis whether the substance posed a risk or not. This has left the ground open for significant divergence between the understandings of different groups. Such divergence is, of course, a principal theme in risk studies.
It has been a major impetus to research on risk perception in particular, and an important conclusion from this has been that lay perception can in some sense be richer than expert analysis (Slovic, 1987). Cultural theories have added to this an understanding that it is cultures which often select risks for particular attention, and which determine the logics we use to react to those risks (Douglas, 1986; Douglas and Wildavsky, 1982; Schwarz and Thompson, 1990). The 'social amplification of risk framework', or SARF (Kasperson et al., 1988), has then provided us with a synthesis of these themes, together with the role of strategic social actors such as the media. It has also provided insights into how secondary or 'ripple' effects are produced when social processes get to work on an issue: the consequences we experience are not just those linked to the

\footnotetext{
* Corresponding author. Tel.: +44 1524594447.

E-mail address: j.s.busby@lancaster.ac.uk (J.S. Busby). 
underlying risk - like toxicity - but those arising from social responses, such as product boycotts, regulation, loss of institutional confidence and so on. Work in the general context of SARF has inter alia helped us understand the arenas in which various social groups operate around a risk issue (for example Renn, 1992), the production and influence of stigmatisation (Kasperson et al., 2005), and the influence of organizational 'recreancy' on the public evaluation of an issue (Freudenberg, 2003).

The case of Deca-BDE, and chemical flame retardants more generally, also illustrates Freudenberg's (1993) thesis about the threatening quality of the division of labour. The risk that flame retardants are meant to mitigate is at least sometimes within the influence of the risk bearer. People who choose to smoke or light candles at home put themselves at risk of domestic fire, for instance. But the risk of toxicity from exposure to flame retardant chemicals, whatever it 'really' is, mostly lies beyond the risk bearer's agency. This creates a division of labour between risk bearers and the various organizations they expect to manage this toxicity risk-the producers of the flame retardants, the formulators who mix these chemicals with others, the textile coaters who apply them to textiles, the furniture manufacturers, retailers, regulators, their supporting scientific laboratories and so on. It might appear to risk bearers that they exchange a risk partly within their control for a risk that causes them to depend on a whole array of organizations, of which nearly all are unknown to them. This dependence may well amplify anxieties in its own right, in a way that appears independent of what emerges from formal processes of risk assessment.

The communications theory metaphor used in SARF has been criticised on various grounds: Bakir's (2005) article and Rosa's (2003) analysis provide a summary of what have been seen as its shortcomings. Perhaps the most significant is the way in which - from the beginning - the framework has been taken to imply that there is some externally given or accurate understanding of risk that is socially amplified or socially attenuated (Rayner, 1988). It is easy to see the problem of such an implication in the Deca-BDE case. Despite the extensive scientific literature on the compound, the question of what kind of risk we 'really' experience in using Deca, and what is the 'real' significance of finding it in various environmental compartments, is too contested to think there is a correct or true pattern of risk. But there is certainly a divergence or disjuncture in understandings of the kind that SARF is about, and these do seem to be the product of social processes rather than random differences of belief. There is also, as in SARF, the production of important secondary consequences beyond direct physical harm. Whether or not the divergence seen in this case could really be said to be an 'amplification' of some less significant discrepancy or problem, we do see in a social response that has somehow become enlarged or expanded beyond what some of the actors (particularly industrial organizations) consider necessary and appropriate.

Given the roots of SARF in communication theory, the most obvious implication for risk managers is to deal with amplification by improving the practice of risk communication. Risk communication in the context of the chemical industry has particularly been linked with acute releases of toxins to communities local to manufacturing sites (for example Chess, 2001). But another heuristic that can be read from SARF is to look for ways of interrupting or impeding the processes that bring about SARF's 'ripple' or secondary effects. It is these secondary effects, like product boycotts or restrictive regulations, that most obviously express what is consequential about heightened risk perceptions. In the case of Deca-BDE the debate in recent years has been strongly oriented around getting regulators to ban the substance and getting users to find substitutes. So it is natural to look at an industry's attempt at averting regulation as an attempt to interrupt a risk amplification process.

Another of the criticisms that has been made of SARF is that it is not a predictive theory (Rayner, 1988). It does not, for instance, predict whether the social reaction to scientific findings about Deca will ultimately lead to regulatory action. But it can still be seen as a model of the mentality that actors adopt when dealing with a risk issue. We may not be able to say objectively whether there is amplification of a true risk level, but from a particular actor's standpoint it is quite likely there is at least some best estimate of a true level, and that there are social phenomena that distort this estimate, and that such distortions have important consequences. So, although SARF may not tell us what is going on in the social world at large, it can serve as a representation of a certain mentality. This notion of risk amplification as a mentality, rather than an objective description of events, also fits with the idea that organizations are not simply stimulated into risk managing actions by their environment but by their perception of the environment (Chess, 2001). Chemical manufacturers with an amplificatory model of a risk issue will perceive the possibility of risk understandings elsewhere becoming amplified and potentially leading to boycotts and regulation in a way that is somehow unjustified or in need of counteraction. If we go along with the idea of SARF as a model of actors' mentalities it cannot then be predictive, since it might lead those actors to behave in such a way as to avoid the outcomes that the model might suggest to them. Yet it can still serve as an organizing principle, illuminating the way in which different actors explain to themselves how anxieties can become inflated, and how this may threaten their interests.

The notion of risk amplification as an actor's way of evaluating the responses of other actors also fits in with the observation that, in cases like that of Deca, there are usually some actors who seem to see the response as being distinctly attenuated rather than amplified. To them the general reaction and the regulatory response looks underplayed and inadequate given the potential seriousness of the risks they see in this substance and its emergence as an environmental contaminant. Their mentality appears to be that negative amplification of a risk has occurred and that this too has secondary effects. Thus, whether the amplification is positive or negative, the basic notion of social amplification provides a way of explaining to one actor how another's responses can seem so inappropriate.

\subsection{A problem of possible toxicity}

The polybrominated diphenyl ethers (or PBDEs) were brought into production to serve an essentially protective function as chemical flame retardants, particularly in the UK where it 
became necessary to meet new legislation aimed at reducing the risk of fire in homes, as well as flammability standards specific to large, institutional product users such as the UK National Health Service. But these compounds then became a risk issue in their own right. Their introduction followed a recent history of other brominated flame retardants that had become embroiled in controversy (for example 'PBBs' and 'TRIS'), and they had appeared in environmental chemists' analyses when they were looking for substances already known to be toxic, such as PCBs, in various environmental compartments. PBDEs became a focus for advocacy groups, such as the WWF, when they were found to be accumulating in marine organisms. They persisted in the environment and were suspected of being bioaccumulative and toxic. A pivotal study appeared to show their concentration in Swedish breast milk increasing exponentially over time (Meironyte et al., 1999), the less-brominated members of the PBDE group were banned in some countries, and they were withdrawn voluntarily from production in others. This left a heavier compound, known as decabromodiphenyl ether, or simply 'Deca', in production.

Eventually this too came under suspicion, and was the subject of a substantial risk assessment (ECB, 2002, 2004) in Europe. Nonetheless, as described in more detail in a later section, this risk assessment seems to have done little to settle the issue. It was unable to find significant positive evidence of risk to human health, yet at the same time concluded that it was 'not possible to say whether or not on a scientific basis there is a current or future risk to the environment' (ECB, 2004). The European member states agreed to a 10 -year programme of continued monitoring and further investigation to reduce the 'unresolved uncertainties'.

Much of the industry that uses Deca believed it had little alternative but to carry on using it, on the basis that alternatives were less effective and more expensive, and even less was known about their health effects. As the EU risk assessment neared completion there had been considerable concern in the industry that Deca would be regulated, would come under marketing restrictions and might even suffer a complete ban. Even if restrictions had been tolerable they might have reinforced the belief that Deca was dangerous in some official sense. There had also been recognition that, for all the uncertainty, it had been discharged to the environment in large quantities over many years. And both advocacy groups and some governments, notably those in Nordic countries, had continued to lobby against it. The objects of their lobbying included large retailers of products containing Deca. The industry's response was a voluntary programme of emissions control, and it is this programme that is the subject of our case study.

The purpose of the study was to find out what is constitutive of this kind of emissions control programme: what qualities appear to have defined it in the eyes of those involved, to have been necessary for its gestation, and to have contributed to its capacity to manage the threat to the production and use of Deca-that is, to act as an 'interruptor' to a risk amplification process. Our approach has been to analyse how actors talk and write about the programme, both in interviews and official publications, in order to draw out these constitutive elements. This should help contribute to our general understanding of why actors caught up in a largescale risk issue act they way they do-in response both to their representations of the underlying, physical phenomena and their representations of other actors.

\section{Method}

A case study method was used (for example Yin, 2003; Stake, 1995; Eisenhardt, 1989), largely because of the inter-connectedness of phenomenon and context. No two cases of what might be called 'risk amplification' are likely to be very similar when the nature of this amplification seems to be so dependent on chance events and historical circumstances. Case study methods are also highly suited to explaining organizational activity through actors' subjective viewpoints (Easterby-Smith et al., 2002). It is these viewpoints that are central when using the idea of risk amplification as a way of characterising people's mentalities, rather than as an objective description of a social process. This particular case study was an 'instrumental' one (Stake, 1995), concentrating on the development of a single programme rather than comparing several programmes of a similar kind. As a result, care is needed in generalizing on the findings, but it provides the opportunity for a relatively deep analysis of a situation that has a widespread importance in the management of chemical contamination.

The data consisted of a set of 15 interviews, of which two were collective (involving three people) and the remainder individual, together with materials given or mentioned to us by the interviewees, including codes of practice, seminar presentations and reports, and briefings. Four of the interviews were with brominated chemical producers or their representatives, two with formulating companies, three with product manufacturers or their representatives, two with retail companies, three with advocacy group representatives and one with a scientist whose work on Deca had been funded by both industry and advocacy groups. The interviews were unstructured, with a broad agenda that covered the subject of the emissions control programme, how it originated, how it has worked, and what its outcomes have been. At least two researchers were involved in each interview, and the data consisted of the notes they took and audio recordings.

Although the theoretical background to the study is provided by work on social risk amplification, the analysis of the data was inductive and grounded, drawing on the spirit although not the procedure of some of the mainstream approaches developed for qualitative analysis (for example Glaser and Strauss, 1967; Schatzman and Strauss, 1973; Strauss and Corbin, 1990). The data was first inspected for references to the emissions control programme in question, known as Voluntary Emissions Control Action Programme ('VECAP'), and these references were very broadly grouped into constitutive qualities-qualities of the programme that appeared to be central to its nature and its functioning given the accounts of the interviewees. Our analysis was not of what interviewees declared in some simple sense defined the programme, but what we inferred to define the programme from the way they chose to describe it. For example, collectivity across an extended supply chain was a 
constitutive quality: the programme was strongly defined by the way in which chemical producers, formulators, coaters and product manufacturers found they needed to act jointly. This was far from being a logical necessity, on face value, because emissions were strongly concentrated at one tier in this supply chain. But it became clear in interviewees' accounts how a pattern of mutual interests and concerns, and a particular distribution of expertise and power, meant that the actors were drawn to making the programme the object of a particular collective group.

We have divided up the account of the case into two sections. The first section describes the case setting: the history of the chemicals in question, and the nature and history of the emissions programme. The section after this presents the analysis of the constitutive elements of the programme.

\section{Case setting}

\subsection{Origins of the programme}

Deca is added to textiles and plastics, in which it is mixed mechanically but does not combine chemically. The focus of our study was textiles application, as it has been particularly difficult for most manufacturers of textile products in the UK at least to find substitutes for Deca in textile coatings. Flame retardants not based on Deca are seen by the industry as being much more limited in their application, thereby restricting the range of available fabrics. They have to be used in such high quantities that they alter the fabric properties and are less effective as a flame retardants. The UK in particular has stringent flammability standards for furnishings, with performance demands that are considered as being difficult to meet with chemicals other than Deca. In contrast to standards in other European countries, for example, they impose a durability demand that stipulates sustained flame retarding performance even after soaking. There are several tiers in the supply chain for treated products. Chemical manufacturers supply Deca to 'formulators', who combine it with binding substances and other coating components, such as dyes. Formulators then supply the mixture to textile coaters. Some of the coaters are essentially contractors to other companies, while others manufacture textile products, such as window blinds, in their own right. Even the commissioned coaters buy their own coatings, however, as their expertise lies in knowing which formulations to buy and how to apply them. Some coated textiles are then supplied to product manufacturers for example manufacturers of furniture - and these supply to retailers.

The 10-year risk assessment of Deca under the EU Existing Substances Regulations, from 1994 to 2004, led to two publications (ECB, 2002, 2004). It was portrayed by one interviewee, a chemical producer, 'as lacking in suspicion as any chemical could be' and 'as close as you can get to nontoxic'. No significant risks to human health were conclusively identified, all safety margins were high, and all ratios of predicted environmental concentration (PEC) to predicted no effect concentration (PNEC) were less than one. But doubts remained about the applicability of the risk assessment methodology to Deca and these, allied to concerns about the substance's environmental and health effects, led the member states to agree to further investigation. Some member states were said to have preferred a ban, on precautionary grounds, instead of continued investigation and the emissions control programme that we describe in this article. Deca had been detected in the environment and in a wide variety of fish and animals, including humans, it had been in production for 38 years, and it was persistent-a quality that was intrinsic to its functioning as a flame retardant. There was also a broad range of 'inconclusive evidence' (ECB, 2004) pointing to potential health hazards. Among the concerns raised in the scientific literature are the potential for Deca to debrominate to form more toxic congeners, both in the environment (Stapleton et al., 2006) and in humans (Frederiksen et al., 2009; Thuresson et al., 2006). There is evidence that it can contribute to neurodevelopmental effects (Viberg et al., 2007), hyperthyroidism (Betts, 2008), and adverse birth outcomes (Chao et al., 2007).

Actors in at least three tiers of the industrial supply chain (chemical producer, coatings formulator and textile coater) freely admitted that emissions of Deca had been unnecessarily high in the past, and that the significance of its persistence had not been fully appreciated. Some have claimed that it was the 'general low toxicity' of brominated flame retardants that 'may have resulted in a lack of attention being paid in the past to emissions to the environment' (Tange et al., 2007). But there was a recognition in the industry that what appeared to some to be a null risk assessment did not amount to the absence of a risk, and certainly not to the absence of a risk issue. It appears that it was the textile coating process that was probably the largest source of emissions within the chain of manufacturing processes. The main problem had been that substantial quantities of waste coating material were washed into the general sewer, extracted by local water company's treatment plants, and typically deposited as sewage sludge on the land. Coatings consisted of Deca with antimony trioxide and acrylic and other polymers to bind it to a fabric, and as this was very viscous it had been impossible to get a complete yield during the coating process. Typically coating paste was left on the application rollers and had to be removed during changeovers. This residual material, in many cases, was then simply washed into the sewer. Some firms claimed to operate a 'dry' process in which they used waste cloths to wipe equipment down, or had a bunded area and their own waste treatment plant. Waste Deca was then encapsulated and removed to controlled landfill sites by licensed contractors. But the textile coaters tended to be very small firms so often lacked treatment facilities. The manufacturers and formulators were much larger, and as discharges to them represented lost profit in a clearer way they were more strictly controlled.

It had therefore appeared to the textile coaters and the formulators that supplied them that Deca might have been about to be banned: 'we were coming to the end of the Deca risk assessment and legislation seemed likely, if not imminent-if we hadn't taken voluntary action it's likely that the outcome of the risk assessment would have been legislation' (a textile coating representative). In the case of textile coatings, more than plastics, there was no obvious substitute for Deca. Informants described searching for replacement 
flame retardants, but the available alternatives would have been needed in such quantities, to meet the regulatory standards, that they would have both been very costly and severely affected the qualities of the textiles they were applied to. For example (from a product manufacturer): 'there are alternative products but they are not as effective either technically or cost wise, but you'll see a lot of material coming out of Sweden about using things like aluminum trihydrate and magnesium hydrate...I have never managed to get aluminum hydrate to work on textile materials without destroying the appearance of the fabric. . you have to put so much into it that you loose a lot of other properties like flexibility and appearance'. And (from a formulator): 'Effective flame retardants that are not based on Deca...would severely limit the range of fabrics which would be available to the public - Deca-antimony systems are very robust - they've allowed the industry to become a fashion item'. As many of the textile coaters were small firms they were also said (by a chemical manufacturer) to be fearful of being included in the scope of Integrated Pollution Prevention Control (IPPC) legislation and 'one-size-fits-all' regulation that imposed similar requirements on firms of all sizes, leading to a disproportionate burden on the smallest.

But the issue of Deca and what would follow the risk assessment concerned the supply chain more generally, not just the textile coaters. One retailer talked about how it had just come through a decade of food scares in the 1990s, how consumers' capacity to trust business had dissipated and how 'everyone was twitchy that [Deca] would be another health scare'. Moreover, the manufacture of Deca had attracted considerable criticism from advocacy groups, who had also contacted retailers. So all organizations in the supply chain were in some sense threatened by the issue-and by the activity of regulators and advocacy groups in particular. The fact that the primary physical problem lay with some very small companies was unfortunate. Precisely because they were small, and several tiers removed from the chemical manufacturers in particular, they seemed not to have realised the significance of simply hosing persistent chemicals into the sewer. They lacked specialised expertise in waste treatment, and they probably saw their operations as being so small as to have no appreciable effect on the world at large. They were also following accepted and legal disposal practices as specified in the safety data sheets for Deca. Thus the problem became how to act collectively across the supply chain in order to deal with emissions that were concentrated somewhere near the middle. This need for collective action, in which many of the participating organizations were having to manage a risk to themselves that originated in emissions elsewhere, led to the formation of VECAP. This was piloted in the UK, but then became a European programme and has now been 'launched' in the USA, Canada and Japan.

The origins of the programme in the UK are probably not accidental. The introduction of stringent flammability standards in the UK followed a spate of some particularly shocking fires and an 'orchestrated campaign' to legislate against highly flammable products. In other countries the pattern of cultural risk selection (Douglas and Wildavsky, 1982; Schwarz and Thompson, 1990) has been different. One of the retail interviewees in another country argued that risk of fire associated with individual behaviour in the house was a matter of individual responsibility, whereas the risk of environmental contamination was of much greater collective significance. The use of foam-filled, fabric-covered furniture is also a practice found in the UK that is not replicated in all other parts of the world. The UK was also seen as a likely source of legislation against Deca, and the UK regulator was the 'rapporteur' responsible for one half of the European risk assessment process. Thus it was in the UK that there was said (by a formulator) to be the particular threat of simultaneously having to meet high flammability standards while suffering restrictions on the use of Deca. It is this that seems to have explained the way in which VECAP originated in the UK, took the UK as a 'pilot', and enjoyed a higher take up in the UK than elsewhere.

\subsection{The nature of the programme}

In terms of what VECAP prescribes to its members, the core is a formal process that consists of six indefinitely repeated steps, 'structured to support the principle of continuous improvement' (quoting a VECAP annual report). The first step is for a firm, described as a 'user', to subscribe formally to VECAP and embed its principles in the firm's procedures. This is followed by a 'self-audit' involving the plotting of production flows that incorporate Deca, and what is called a 'mass balance' calculation that essentially means accounting for all Deca entering the process in terms of its final destination, whether in a product or in waste streams. Uncontrolled emissions are determined by the residue of this calculation. This procedure reflects the difficulty of measuring Deca in effluent directly: it is not a constant stream and so is difficult to sample sensibly and at a reasonable, proportionate cost, particularly given the small size of many of the firms concerned. There is then a 'baseline emission survey' in which the mass balance results are used as a basis for future monitoring. The next stage is to develop a plan to reduce emissions, and the final stage is to put this into practice-and evaluate the consequences to look for further opportunities for improvement.

In many respects, VECAP reflects a wider institutionalisation of the notion of environmental management systems and their formulation in international standards such as the ISO 14000 series-and an associated trend towards self-regulation of environmental performance (for example Anton et al., 2003; Stenzel, 2000). VECAP shares with these approaches a common motivation, namely to act as an alternative to traditional, externalised command-and-control forms of environmental regulation. There are also clear similarities in form: in the toplevel commitment required, the systematisation via formal procedures, the emphasis on monitoring, and the exhortations towards continuous improvement. But it is specifically concerned with a certain compound and prescribes specific, technical measures: 'ISO 14001...is all about the quality of the procedures employed within an environmental management system - not directed at an actual compound or chemicalwhereas VECAP is more about quantifying the actual releases of a specific compound' (a chemical producer). Nonetheless, as we describe later, the promotion of VECAP associates it with ISO 14001, and in subsequent sections we refer to what some of the informants in the study thought about this association. 
The paraphernalia of VECAP includes a Code of Good Practice, a Self-Audit Guidance Document, a Process Flow Chart and a Mass Balance Sheet. The code of practice covers tasks such as emptying of packages, where the aim is both to minimise losses, for example coating matter left in bags, and minimise the possibility of matter being washed to sewer. The know-how incorporated in these practices looks straightforward, involving what amounts to good housekeeping, but it applies to operations that are so prosaic that they might well be performed heedlessly in practice (according to a chemical producer). Within the programme there is also an independent 'product steward' - an individual who is a qualified ISO 14001 auditor - to oversee the VECAP process. Presentations given by representatives of the industry also refer to a 'VECAP Competence Centre (under development)'. Although it remains a relatively small undertaking, the programme has a distinct, independent existence beyond the mere coming together of a handful of organizations.

The outcome looks like a considerable reduction in reported emissions. The programme's first annual report claimed a 75\% reduction in Deca-BDE emissions to water by the UK textiles industry, and 'significant progress in the other EU Member States, on target to cover $90 \%$ of Deca-BDE usage in the EU by June 2007'. One of the interviewees (a textile coaters' representative), and the annual report, stated that some firms had enjoyed a $90 \%$ reduction in emissions. Another outcome has been that most regulators have not banned Deca, or introduced marketing restrictions, and some have been reported as giving highly favourable testimony on VECAP. An EC official was quoted in the annual report as welcoming the programme and supporting voluntary action where this can 'result in environmental improvement hand in hand with economic development'. The UK environmental regulator was reported as being 'pleased to see chemical producers working so effectively with their downstream users to introduce a process to monitor and control emissions. Had VECAP been a regulatory measure, with overall UK emission reductions of $75 \%$ in its first year, it would have been hailed as a major success. That it was achieved as an industry voluntary commitment means that this level of environmental protection was attained far faster and with great savings'. A Dutch government official was similarly quoted in the second annual report as calling the $75 \%$ reduction 'remarkable', and saying that 'Such an initiative deserves to be copied by other industry sectors with other substances'. As we describe later, not everyone felt the programme was so laudable.

\section{Constitutive elements}

In this section we describe what emerged, in our analysis, as being the constitutive elements of VECAP that particularly fitted the idea of VECAP as interrupting a process of risk amplification.

\subsection{Side-benefits and improvement}

An important aspect of VECAP is that it leads to a general process of improvement in the firms that subscribe to it. Its physical consequences are not simply the reduction of Deca flows to the environment, particularly to the sewer, but a reduction in wasted coating compound and an increased awareness of losses in the manufacturing process. One interviewee (a formulator) claimed to be 'amazed' how little some of the companies knew of their own processes. These companies were said to be surprised that they were 'throwing away material', and prior to VECAP had (somewhat ironically) even taken pride in recycling packaging by washing Deca out into the drain. The emphasis in VECAP on good housekeeping, and the idea of plotting material flows, also promoted the view that it was a way of obtaining general improvements. Presentations on VECAP used the rhetoric of 'continuous improvement' that has become integral to concepts of quality management and, as we discuss later, the programme has been linked to general ISO standards. Even the labelling of those who subscribe to VECAP as 'users', and VECAP's prescribed methods as a 'toolkit', indicates we are meant to see VECAP as a tool to some useful end, not merely an exercise in compliance.

The notion of a programme that produces side-benefits for its subscribers as individual firms seemed important to making VECAP attractive to potential subscribers. Some interviewees talked about the particular difficulty of persuading firms partway down the supply chain, such as textile coaters, of the need for controls. Once the risk assessment of Deca had been portrayed as being favourable, it could hardly be called a 'dangerous' compound, so in the textile coaters' eyes did not seem to warrant the attention it was receiving. The threat of regulation provided one reason to join VECAP irrespective of how dangerous Deca was. But averting this threat was a collective good not an individual one, so that there could then have been a problem with free-riding-with firms wanting to obtain the general benefit of avoiding damaging regulation without incurring the individual cost of subscribing to VECAP. The presence of individual sidebenefits helped reduce this danger.

In practice, these side-benefits were taken to different degrees by different companies. Whereas some seem to have benefited from process savings, others (for example a product manufacturer that operated a coating process) already had a strong efficiency programme in which waste and losses were closely tracked. Moreover, some had effluent treatment plants so were not discharging to the sewer at all, only removing Deca as encapsulated solid to controlled landfill. But - while this meant such firms benefited less from VECAP - it also meant the costs of complying with VECAP were limited: only a small amount of additional surveying and monitoring.

\subsection{Trust, collectivity and voluntariness}

Interviewees often talked about responding to the threat of regulation as a specifically collective action. Two for example (a product manufacturer and textile coater's representative) said that at the time the risk assessment was about to published there was a general feeling of the 'need to form a trade association' of Deca users. To some extent there had already been a distinctly collective element in the industry. One interviewee (a formulator) for example argued that 'everyone knew and trusted each other'. But this referred only to the relationships between formulators and coaters. As 
we indicated earlier, the coaters were thought to be a primary source of emissions, yet the controversy surrounding Deca involved the whole supply chain, from chemical manufacturers through to consumer goods retailers. So, although the underlying issue implicated a particular group of firms, the secondary or 'ripple' effects in social amplification terms would have affected all these groups. At this level, there had been little that was collective prior to VECAP. Some interviewees (a chemical producer and formulator) referred to the way in which chemical manufacturers had formerly had little to do with users of their products, who were generally only indirect customers. Another (a former chemical producer) was critical of how chemical producers had formerly paid scant attention to the needs of their customer chain, and another (a retailer) talked about needing to 'learn a common language' and shift from being commercial adversaries to having mutual respect. There were also obstacles to collective activity in the form of anti-trust rules. One interviewee (a chemical producer), for example, said that 'if you look at VECAP overall, I don't know the exact number of people involved. . .that's something that we tend to keep fairly confidential, because of competition law, there are three major suppliers of brominated flame retardants in Europe. . .but we have to be very careful in not exchanging customer information and various other things...so all our things are done through a sanitised way of working forward'. Thus VECAP was not simply a product of existing relationships but a creator of new ones. Reports in praise of VECAP (Tange et al., 2007) in fact say that 'VECAP has succeeded in bringing downstream users, including SMEs together with suppliers of chemical substances.... It has highlighted the importance of communicating through the supply chain...'.

There is a particular respect in which VECAP depends on trust within this collective group. The VECAP process involves an inventory of where Deca is coming from and where it is going to, so textile coaters need to know how much Deca they are acquiring from formulators. However, traditionally the proportion of Deca in the coating formulation has been commercially confidential. Deca is not the only component, and (according to a formulator) it is a matter of technical expertise how much Deca is needed to meet flammability standards, together with binding compounds and other substances. If a textile coater were using several of a formulator's products, and they were told how much Deca they consumed in total, they would not be able to work out the proportion of Deca in each product. But if they were using only one they would. Various interviewees (two formulators and a textile coaters' representative); therefore referred to the need for 'trust' within the VECAP programme. In fact the higher rates of adoption of VECAP in the UK compared with mainland Europe was attributed to the fact that the UK textile coating industry was relatively small, which made it easier for the firms to develop the trust needed to share data of this kind.

The programme was also voluntary. There was an existing collective body in the UK textile coating industry - the Textile Finishers Association - and they were an early locus of VECAP activity (according to two of the formulators and a textile coaters' representative). But the Association's members were not coerced into membership of VECAP, and the stress placed on the individual benefits for these firms reflected the need to sell VECAP to firms rather than impose it. One interviewee (a chemical producer) argued that voluntarism naturally suited a problem like that of Deca emissions where the risk is unclear and disputed, and therefore where the science is sufficiently ambiguous to stall regulatory action. Another interviewee (also a chemical producer) voiced some frustration that his industrial competitors seemed to get away violating regulatory bansundermining the notion that voluntarism necessarily produces less compliance than regulation. A retail interviewee similarly claimed if a retailer is sufficiently embarrassed on an issue with the public the resulting changes it imposes on its supply chain are much more significant than those produced by regulation.

It could be argued that this voluntarism is illusory, given that VECAP was a self-interested response to a regulatory threat, and perhaps in some way an inevitable one. But it was still a choice, its design was in the hands of the industry, and its promoters still had to convince potential members of its worth. It may have been a self-interested action, and it may have followed or arisen in anticipation of specific events, but this does not make it involuntary or forced. Whether voluntarism is objectively more effective than regulation, and whether in fact it fits highly uncertain situations, is hard to assess from this single case. But it was a central quality of the programme in the eyes of its promoters: the programme was something its members could be credited with, and equally be held accountable for-a product of their agency rather than some other group's.

\subsection{Criticisability and criticism}

VECAP has been criticised, for how it works and what it achieves, both from within the programme and from outside. The outsiders' criticism came particularly from advocacy groups, in press reports and briefing material (WWF, 2005), as well as interviews. VECAP was seen as a purely defensive reaction to the threat of regulation, and this raised the question as to whether it could be called an 'initiative' in any meaningful sense. It was also seen by one advocacy group interviewee as 'totally inadequate in that significant exposure will result from the use of articles and not just from Deca-BDE released from the factory'. Another suggested that much of the reduction in emissions claimed for VECAP may have been down to plant closures that were independent of the programme. But even if VECAP were the cause of emission reductions, this should not be the reason for significant approval: 'To stop people washing huge quantities of brominated waste down the sink from textile processing. . to stop that kind of appalling practice, I don't see it as a great step forward by the industry'.

The insiders' criticism was partly directed at the way some saw the programme as having been initiated by the formulators and textile coating industry, but then taken over by the chemical manufacturers and directed at their own ends. There was also a criticism of the basic logic, relying on accounting for a 'mass balance' to determine how much Deca was being emitted by a given firm. This criticism was not particularly hostile in the way that the external criticism was, but it came from authoritative sources and needs to be taken seriously.

The criticism that VECAP neglected emissions beyond the manufacturing process drew the response (from a chemical 
producers' representative) that its goal was to exercise product 'stewardship', generally, and that the current restriction to manufacturing was a temporary matter of trying to 'walk before you run'. The sense was that exercising stewardship across the whole lifecycle, all at once, was an unrealistic ambition. The criticism of VECAP's limited scope has a strong justification: VECAP's basic methods really only suit the manufacturing process and would require a quite different logic to work in the context of product use and disposal. And there is a literature suggesting the main exposure of humans to Deca can come from indoor dust derived from Deca-treated products, not from emissions arising from manufacturing processes (Betts, 2008; Lorber, 2008). Yet it sounds reasonable to argue that a programme of any kind takes time to fully develop. And the programme has been extended to the handling and disposal of packaging and emissions associated with fugitive chemical losses from warehouse storage facilities (according to a VECAP annual report). This is a small extension but can be pointed to as a recognition of the limitations of concentrating on manufacturing emissions. As for the criticisms, from another advocacy group, that VECAP was 'only' a response to the threat of regulation, and deserves no praise for doing what always should have been done, the obvious defence is that this does not reflect on the effectiveness of VECAP (whether this is significant or not) in reducing emissions. The critics' concern might be that what they see as VECAP's limited accomplishments could be interpreted more widely as having addressed all outstanding concerns relating to Deca-and that this might reduce the political incentive to address issues outstanding from the risk assessment, and perhaps ban Deca. But the obvious response is that VECAP's limited ambitions are in some way proportionate to an inconclusive risk assessment, and that it does not logically preclude further action as further evidence emerges.

These responses do not rebut the criticisms in some definitive way, but they look plausible to an uncommitted audience. What we can see in VECAP, therefore, is an action that attracts criticism because it sustains the idea of an industry continuing to use a suspected substance-yet it provides a basis for responding to the criticism in a way that does not fit a caricature of a malign industry. It is criticisable, so engages those actors who are opposed to Deca, but the criticisms of VECAP appear to be answerable. Generally, a programme such as VECAP appears to provide a 'lightning conductor': a way for an industry, or network of organizations, to take a defensible line on a risk issue. If critics can be persuaded to direct their attacks at VECAP the industry has a more convincing story to tell the world than it would if the attacks were directed solely at the main activity of producing and using Deca.

\subsection{Independence and institutionalisation}

VECAP has been institutionalised and given an independent status in several ways. One is that it has explicit aims and processes, together with various paraphernalia like codes of practice. Another is that there is a formal appointment of an auditor within the programme, whose 'independence' is regularly stressed by those talking about VECAP. There are said to be plans for VECAP 'certification'. And VECAP is explicitly linked with international standards: 'VECAP is based on recognised environmental management programmes such as 14001 and EMAS but stripped down to basics so that it really focuses on the target chemical and can be implemented by Small and Medium enterprises'. In the interviews there was more ambivalence about the association with ISO 14001, one interviewee (a textile coaters' representative) making the point that VECAP was about demonstrably reducing emissions, not merely operating a management system. But the association helps to institutionalise VECAP.

All this seemed necessary for several reasons. The first is that, as indicated earlier, the firms with the greatest emissions were those (according to a formulator) that did not have the resources to 'think it out for themselves'. An explicit, systematic, codified approach was needed to support them, and be seen to support them. The second reason for institutionalisation was credibility, in the eyes of regulators particularly but also other players such as retailers who stressed the environmental credentials of their product lines. One retailer said that if VECAP had not existed it would have been more likely to move towards substituting Deca. Institutionalisation seemed necessary to assure actors that the programme would continue as long as it was needed, with sufficient resources, imposing sufficient obligations on its members.

Third, the programme was also the source of the data that demonstrated it worked. Its constitution and processes needed to be institutionalised enough that people believed the data it produced. One interviewee (a regulator) talked about how the UK Textile Finishers' Association became a credible 'expert' source of information in dealings with the Government because VECAP meant it could somehow validate the figures it was giving. Data from the 'mass balance' exercises went to the UK Department for Environment, Food and Rural affairs. Although they were not put into the public domain directly, they were used for the EU Risk Assessment biennial updates. Some of the programme's critics saw this as problematic, one (an advocacy group representative) saying 'we're entirely reliant on what they're telling us and that's never a comfortable position to be in'. But the more formalized and institutionalised the process the easier it was to deal with such criticism.

Fourth, in particular regard to the activity of advocacy groups, the programme needed to have a sufficient weight in the wider debate that was going on about synthetic chemicals, the natural environment and human health. Given that commercial corporations are constituted to pursue private, or at least limited, interests, it is natural that people will want to see benevolent behaviour institutionalised to believe that it amounts to anything. Institutionalisation helps avoid the notion that, as a creation of a particular industry, the programme is also their creature. A particular issue in the Deca case is the association of the chemical producer's umbrella group (the 'Bromine Science and Environmental Forum', BSEF) with the public relations firm Burson-Marsteller, and the attendant criticism from, for example, Corporate Europe Observatory (CEO, 2005). Whether the institutionalisation of VECAP helps the programme escape the scepticism accorded to BSEF is unclear. 
Finally, there was a sense that the problem for the industry was not a temporary one of trying to win a debate and being able to move on. One interviewee (a chemical producers' representative) argued that 'environmental groups may change tack but will never lose interest-it's imprinted in their mind that bromine compounds are a bad thing'. The expectation was that the industry's use of this compound, and related ones would always be contested, and that what was needed was not a transitory project but an established, indefinite programme. Moreover, Deca was seen (by a former chemical producers' representative) as being a 'line in the sand'. He argued that production volumes of Deca were small compared with other chemicals that could, if the industry abandoned Deca, become the focus of hostile debate.

\subsection{Takeover, labelling and branding}

The VECAP programme, as well as becoming institutionalised, appears to have been taken over from the formulators and textile coaters by the chemical manufacturers, extended by them into new domains, and given a distinctive 'branding'. We referred earlier to the path of VECAP's expansion. It appeared to originate in discussions among textile finishers and formulators, 'coming to light' before the risk assessment was finished when Deca was under threat of a ban in 2003 (according to a textile coaters' representative). A particular individual in one of the formulating firms had mooted the notion of a voluntary scheme in a risk assessment meeting. But the programme came to be 'formalized' and perhaps dominated by the chemical producers. One interviewee talked about the programme being 'taken over by the bromine industry who did not seem to fully understand its particular roots in the textile industry'. Since they supplied to the plastics industry in parallel with the textiles industry these chemical manufacturers also expanded the scope of the programme to cover plastics as well. Presentations by chemical industry representatives say that it was the three manufacturers of Deca supplying to Europe (two American and one Israeli) who 'cooperated to develop the VECAP programme'.

Some of the promotion of VECAP looks inflated. We described earlier how it has been described as a programme of 'product stewardship', and one case was described to us of a chemical manufacturer declining to supply a specific customer because it refused to subscribe to VECAP. But the chemical manufacturers have not generally withdrawn sales to firms refusing to join VECAP. And VECAP's restriction to emissions in the manufacturing process, however transient, contradicts the general notion of stewardship. The findings of highly elevated levels of Deca in dismantling workers (Bi et al., 2007), and the concern of some manufacturers about the exposures of product users to Deca, indicate that this is a significant concern. Nonetheless, the takeover and extension of VECAP, and its branding, all seem basic to the purpose of influencing the world. Simply controlling emissions is unlikely to gain much attention in a risk issue that is so contested, particularly when the risk assessment process has failed to resolve the disparity of opinion. So there is a sense in which the industry will naturally want to influence people's view of their credentials as risk managers in ways which magnify the impact of the physical accomplishments that have come from
VECAP. Larger corporations such as the chemical manufacturers tend to have more representation in bureaucratic processes than smaller firms, so a 'takeover' by them may be necessary for a programme such as this to achieve sufficient voice in regulatory thinking and regulatory dialogue. It may be construed that the larger corporations at both ends of the supply chain - large chemical producers and large retailers are in some way exploiting VECAP, but it is necessary to co-opt powerful players.

The branding of the programme, and its association with ideas of 'stewardship', also seem necessary in overcoming the hurdles to the acceptance of a voluntary programme. As work on trust in relation to risk indicates (Lang and Hallman, 2005), people have particularly low levels of trust in industry. There is of course a danger that an over-concentration on mere publicity will be counter-productive, but at the same time it seems necessary to find a way of encapsulating the mentality behind VECAP in a communicable form. Members of the programme presented themselves as taking a new approach to dealing with public concern; claiming VECAP as a good example of how a sector 'looked at the future and decided it had got to change'. Interviewees in the industry talked several times about having a general idea of being concerned with emissions beyond their own organizational boundaries, of reducing emissions whether or not the evidence of harm was contentious, and of being 'pro-active' in finding and controlling hitherto unknown sources of emissions. But this change of mentality is hard to make visible in an arena that is heavily politicised and contested. And, because controlling emissions in an industry like this is a matter of taking local actions in many places, it is hard to generate the impression of industry acting collectively with a common goal in mind. Having a labelled, branded programme helps to make the idea of an industry that has made a qualitative change in its thinking and behaviour a clearer notion to the world at large. It is a vehicle for self-portrayal as a group of proactive, precautionary and responsible actors.

\section{Discussion}

The Deca case is particularly interesting because the contaminant in question is itself a risk controlling measure, and much of the discourse reflects the 'balancing' of the toxicity risk from using the compounds with the fire risk from not using them. Yet there is a strong divergence in the understandings of different actors-notably industry and advocacy groups but also among governments. From their individual standpoints, the responses of other actors look like social risk amplification: responses that are somehow amplified (positively or negatively) and therefore disproportionate to the risk signals or evidence that produce them. From the industry's standpoint, in particular, there appears to be a level of controversy that belies a risk assessment that could find little positive evidence of significant risk. Moreover, as models of social risk amplification emphasise, this controversy has secondary outcomes beyond the merely physical risks in question: for example the possibility of bans on chemical production. The actions taken by industry look like a way of interrupting the generation of these secondary outcomes. 
At a more detailed level, the way industry has gone about this 'interruption' also fits with our ideas about the social amplification of risk:

- Institutionalisation was about counter-acting amplification stages, convincing actors who could produce the next stage of response - particularly regulation - not to produce this response. It was a way of overcoming the problem that any effort at risk management that is not organized, collective and systematic is an effort that is suspected of being ineffectual and half-hearted. We depend on institutions and organizations, not haphazard activities by transient bodies, to manage risk-as can be seen in the particularly strong risk amplification that seems to accompany organizational 'recreancy' (Freudenberg, 2003).

- Criticisability was about acting in a way that could be criticised, by actors both within and outside the programme, yet provide a defensible and reasonably robust basis that was not completely undermined by this criticism. The metaphor of a 'lightning conductor' was suggested, to add to SARF's own metaphor of an amplificatory communications system. Lightning conductors, in some loose way, should reduce amplification effects. So, while VECAP can be criticised for failing to deal with the exposures of product users to Deca, it can be defended for dealing directly with the emissions from the manufacturing process that are thought to explain the detection of Deca in various environmental compartments.

- Side-benefits were said to be a way of convincing members of a collective effort to subscribe to collective action and to avoid free-riding. It was a whole network of organizations that were jointly involved in making and using Deca, and most, if not all, needed to participate in the effort if it was to be convincing as an effective attempt to manage a risk. Society at large has a sense that some risk issues belong to collections of organizations, not merely organizations as individual entities, so the failure to act collectively would be seen as flawed and lacking in seriousness.

- The takeover and branding of the programme was again about producing a signal that would counteract the otherwise amplificatory influence of hostile publicity. VECAP seemed to need a base in a substantial industrial grouping, involving relatively large firms, and it needed a coherent way of communicating what were otherwise a set of simple, minor and rather pedestrian activities-like improving the housekeeping task in small textiles coating firms.

In controversies like that surrounding Deca, the industry finds itself in a contradiction. It denies that its activity in producing and using Deca is dangerous, it broadly needs to carry on denying this in order to avoid the charge of irresponsibility or 'recreancy', and anyway the Europe risk assessment could be portrayed as supporting this. At the same time it has concluded it must act to reduce the emissions that it denies are dangerous, and thus appears to take the possibility of danger seriously. But having a concept of risk amplification, or something similar, helps avoid this contradiction, because it is then possible to believe that a risk is minor, yet also to believe that other actors can come to believe the same risk is major, and make demands that need to be acknowledged to avoid punitive outcomes. The particular framework of social risk amplification is not the only one that captures this idea, and alternatives like Kuran and Sunstein's (1999) model of availability cascades also look applicable. Actors like the NGOs, and the industry group responsible for the VECAP programme, look distinctly like Kuran and Sunstein's 'availability entrepreneurs' for instance. But SARF, because it is too loose to be a predictive theory, also accommodates the idea that actors within a process of amplification can have some sense of being in such a process, and act in a way that reflects this and seeks to avoid the outcomes that they envisage. And, while we can criticise SARF on the basis that it is seems to imply that there is some externally given, or true, level of risk that subsequently becomes amplified, this does in fact capture something of the mentality of the actors in cases like this. The industrialists and their critics do talk as though there is a real risk that becomes amplified or attenuated in the course of society's dealings with it.

Whether it is desirable that such amplification processes should be interrupted - by building programmes like VECAP - is as much a matter of debate as the underlying risk issue. The advantage of having a notion of social risk amplification as an objective statement about the world is that we can readily say the social response to risk information has become disproportionate, and is probably undesirable, so any process that interrupts the amplification process is broadly beneficial. But once social risk amplification becomes a way of describing one group's views of other groups there is no reason to make this inference. In this instance, while industry representatives could read the European risk assessment as being favourable because it identified no 'risks', others - including a representative of the regulatory body that participated in the risk assessment - argued that 'serious concerns remain about Deca-BDE's long-term environmental (and health) impacts'. So exactly what risk 'signal' is being amplified in a social amplification process is not agreed upon. This notion that social amplification is in the eye of the beholder, rather than an objective phenomenon, then means that the benefits of managing risk amplification become as much a matter of perspective as amplification itself. The amplification of a given risk signal could be seen as being beneficial in a situation where initial risk information is biased in such a way as to underplay risks, for example in the case of a substance whose effects tend to be overlooked under conventional testing protocols, or whose harmfulness only becomes evident over extended periods. Amplification could also be seen as a way of re-introducing legitimate social concerns like equity and voluntariness when these become lost in technical risk assessments. Moreover, the relevant groups participating in and observing social risk amplification change over time. In cases like that of chemical flame retardants, restriction of one compound is likely to lead to bigger markets for alternative compounds, and one chemical producer's loss might be another's gain (or even their own gain). Thus it is highly dependent on context exactly who sees risk as being amplified and who stands to gain from interrupting the risk amplification process. 


\section{Conclusion}

There has been a long-standing dispute over the risks associated with PBDEs, their appearance in various environmental compartments and the toxicity risks associated with human exposure to them. In this article we reported a study of how an industry had developed a collective programme to control emissions of one particular compound-a compound whose risks seem to be beyond the capacity of the existing regulatory and scientific establishment to pin down, and which continues to be surrounded in controversy. This programme was linked to the notion of interrupting a process of social risk amplification. The risk amplification model has been criticised for its lack of predictive credentials, but it does appear to express the mentality of the actors in the case of Deca. We can see the interpretations and actions of industrial actors, in particular, as being based on the idea of a minor risk of toxicity that has somehow become amplified through social discourse into a major threat, likely to lead to the secondary effect of regulation. But it became possible, in their eyes, to interrupt the amplification process and avert regulation by enacting a programme of voluntary emission controls. This programme, to be credible in the view of both insiders and outsiders, had to be institutionalised, it had to confer sidebenefits on its participants, it had to be strongly branded and it had to be capable of being criticised. The programme has been associated with considerable reductions in emissions of the flame retardant compound at which it was aimed, although the extent to which it caused these reductions is disputed among the actors, and the programme does not address the considerably greater end-oflife emissions of Deca.

The findings from the study are naturally limited by its small scale and qualitative methodology. But what we learn from it is that actors - particularly industrial actors sometimes have to simultaneously uphold the view that a contaminant is not significantly hazardous and act as though it were hazardous: the way they then go about acting fits the idea that they think in terms that closely resemble the social amplification of risk framework.

\section{Acknowledgements}

This work was partly funded by grant F/00 185/P from The Leverhulme Trust. We are very grateful to all who helped arrange and participate in the interviews, many of whom gave valuable feedback on an earlier draft of this article. Finally, many thanks are also due to the reviewers of a previous version of the manuscript. The views expressed here are solely those of the authors.

\section{R E F E R E N C E S}

Anton, W.R.Q., Deltas, G., Khanna, M., 2003. Incentives for environmental self-regulation and implications for environmental performance. Journal of Environmental Economics and Management 48, 632-654.
Bakir, V., 2005. Greenpeace v. Shell: Media exploitation and the Social Amplification of Risk Framework (SARF). Journal of Risk Research 8, 679-691.

Betts, K., 2008. Unwelcome guests-PBDEs in house dust. Environmental Health Perspectives 116, A202-A208.

Bi, X., Thomas, G., Jones, K.C., Qu, W., Sheng, G., Martin, F.L., Fu, J., 2007. Exposure of electronics dismantling workers to polybrominated diphenyl ethers, polychlorinated biphenyls, and organochlorine pesticides in South China. Environmental Science and Technology 41, 5647-5653.

CEO, 2005. House of Mirrors. Burson-Marsteller Brussels lobbying for the bromine industry. Corporate Europe Observatory, January 2005. Last accessed at http:// www.corporateeurope.org/lobbycracy/houseofmirrors.html on $14 / 10 / 08$.

Chao, H.R., Wang, S.L., Lee, W.J., Wang, W.F., Päpke, O., 2007. Levels of polylbrominated diphenyl ethers (PBDEs) in breast milk from central Taiwan and their relationship to infant birth outcome and maternal menstruation effects. Environment International 33, 239-245.

Chess, C., 2001. Organizational theory and the stages of risk communication. Risk Analysis 21, 179-188.

Douglas, M., 1986. Risk Acceptability According to the Social Sciences. Routledge and Kegan Paul, London.

Douglas, M., Wildavsky, A., 1982. Risk and Culture. An Essay on the Selection of Technological and Environmental Dangers. University of California Press, Berkeley.

Easterby-Smith, M., Thorpe, R., Lowe, A., 2002. Management Research: An Introduction, 2 ed. SAGE, London.

ECB, 2002. European Union Risk Assessment Report: Bis(pentabromodiphenyl ether), 1st priority list, Volume 17, EUR 20402 EN. European Chemicals Bureau, Institute of Health and Consumer Protection, European Commission.

ECB, 2004. Update of the Risk Assessment of Bis(pentabromodiphenyl ether). R013_0405_env, Final Environmental Draft of May 2004, European Chemicals Bureau.

Eisenhardt, K.M., 1989. Building theories from case study research. Academy of Management Review 14, 532-550.

Frederiksen, M., Vorkamp, K., Thomsen, M., Knudsen, L.E., 2009. Human internal and external exposure to PBDEs-a review of levels and sources. International Journal of Hygiene and Environmental Health 212, 109-134.

Freudenberg, W.R., 1993. Risk and recreancy: Weber, the division of labour, and the rationality of risk perceptions. Social Forces 71, 909-932.

Freudenberg, W.R., 2003. Institutional failure and the organizational amplification of risk: the need for a closer look. In: Pidgeon, N., Kasperson, R.E., Slovic, P. (Eds.), The Social Amplification of Risk. Cambridge University Press, Cambridge, UK, pp. 102-120.

Glaser, B.G., Strauss, A.L., 1967. The Discovery of Grounded Theory. Aldine Publishing Company, New York.

Kasperson, R.E., Renn, O., Slovic, P., Brown, H.S., Emel, J., Goble, R., Kasperson, J.X., Ratick, S., 1988. The social amplification of risk: a conceptual framework. Risk Analysis 8, 177-187.

Kasperson, R.E., Jhaveri, H., Kasperson, J.X., 2005. Stigma and the social amplification of risk: towards a framework of analysis. In: Kasperson, J.X., Kasperson, R.E. (Eds.), The Social Contours of Risk, vol. 1. Earthscan, London, pp. 161-180.

Kuran, T., Sunstein, C.R., 1999. Availability cascades and risk regulation. Stanford Law Review 51, 683-768.

Lang, J.T., Hallman, W.K., 2005. Who does the public trust? The case of genetically modified food in the United States. Risk Analysis 25, 1241-1252. 
Lorber, M., 2008. Exposure of Americans to polybrominated diphenyl ethers. Journal of Exposure Science and Environmental Epidemiology 18, 2-19.

Meironyte, D., Bergman, A., Noren, K., 1999. Polybrominated diphenyl ethers in Swedish human milk. A time related trend study, 1972-1997. Journal of Toxicology and Environmental Health Part A 58, 101-118.

Rayner, S., 1988. Muddling through metaphors to maturity: a commentary on Kasperson et al. 'The Social Amplification of Risk' Risk Analysis 8, 201-204.

Renn, O., 1992. The social arena concept of risk debates. In: Krimsky, S., Golding, D. (Eds.), Social Theories of Risk. Praeger, Westport, CT, pp. 179-196.

Rosa, E.A., 2003. The logical structure of the Social Amplification of Risk Framework (SARF): metatheoretical implications and policy implications. In: Pidgeon, N., Kasperson, R.E., Slovic, P. (Eds.), The Social Amplification of Risk. Cambridge University Press, Cambridge, UK, pp. 47-79.

Schatzman, L., Strauss, A.L., 1973. Field Research. Prentice-Hall, Englewood Cliffs, NJ.

Schwarz, M., Thompson, M., 1990. Divided We Stand: Redefining Politics, Technology and Social Choice. University of Pennsylvania Press, Philadelphia.

Slovic, P., 1987. Perception of risk. Science 236, 280-285.

Stake, R.E., 1995. The Art of Case Study Research. Sage, Thousand Oaks.

Stapleton, H.M., Brazil, B., Holbrook, R.D., Mitchelmore, C.L., Benedict, R., Konstantinov, A., Potter, D., 2006. In vivo and in vitro debromination of decabromodiphenyl ether (BDE 209) by juvenile rainbow trout and common carp. Environmental Science and Technology 40, 4653-4658.

Stenzel, P.L., 2000. Can the ISO 14000 series environmental management standards provide a viable alternative to government regulation? American Business Law Journal 37, 237-299.
Strauss, A., Corbin, J., 1990. Basics of Qualitative Research. Sage Publications, Newbury Park, CA.

Tange, L., Buszard, D., Steukers, V., Adriaenssens, P., 2007. VECAP: controlling emissions protecting the environment by continuous improvement. In: Fourth International Workshop on Brominated Flame Retardants, Amsterdam, 24-27 April.

Thuresson, K., Höglund, P., Hagmar, L., Sjödin, A., Bergman, Å., Jalobsson, K., 2006. Apparent half-lives of hepta- to decabrominated diphenyl ethers in human serum as determined in occupationally exposed workers. Environmental Health Perspectives 114, 176-181.

Viberg, H., Fredriksson, A., Eriksson, P., 2007. Changes in spontaneous behaviour and altered response to nicotine in the adult rat, after neonatal exposure to the brominated flame retardant decabrominated diphenyl ether (PBDE 209). Neurotoxicology 28, 136-142.

WWF, 2005. WWF's Recommendations for 1. REACH 2.In: Action on Deca-BDE, WWF's Briefing for Competent Authorities, Helsinki, June.

Yin, R.K., 2003. Case Study Research: Design and Methods, 3rd ed. Sage, Thousand Oaks, CA.

Jerry Busby is a senior lecturer in the Lancaster University Management School. His research interests are in risk analysis, risk management, organizational failure and organizational reliability.

Ruth Alcock is a senior research fellow in the Lancaster University Management School. Her research interests are in chemical risk management, having formerly worked as an environmental chemist.

Brian MacGillivray is a research assistant in the Lancaster University Management School. His interests are in the governance of risk. 